\title{
The compactness of the sum of weighted composition operators on the ball algebra
}

\author{
Ce-Zhong Tong and Ze-Hua Zhou*
}

* Correspondence:

zehuazhou2003@yahoo.com.cn

Department of Mathematics,

Tianjin University, Tianjin, 300072

People's Republic of China

\begin{abstract}
In this paper, we investigate the compactness of the sum of weighted composition operators on the unit ball algebra, and give the characterization of compact differences of two weighted composition operators on the ball algebra. The connectness of the topological space consisting of non-zero weighted composition operators on the unit ball algebra is also studied.

2000 Mathematics Subject Classification. Primary: 47B33; Secondary: 47B38, 46E15, $32 \mathrm{~A} 36$.
\end{abstract}

Keywords: Weighted composition operator, Ball algebra, Sum, Difference, Inequality

\section{Introduction}

Let $H\left(B_{N}\right)$ be the class of all holomorphic functions on $B_{N}$ and $S\left(B_{N}\right)$, the collection of all holomorphic self mappings of $B_{N}$, where $B_{N}$ is the unit ball in the $N$-dimensional complex space $\mathbb{C}^{N}$. The closure of $B_{N}$ will be written as $\overline{B_{N}}$. Denote by $A=A\left(B_{N}\right)$, the unit ball algebra of all continuous functions on $\overline{B_{N}}$ that are holomorphic on $B_{N}$. Then $A$ is the Banach algebra with the supremum norm

$$
\|f\|_{\infty}=\sup \left\{|f(z)|: z \in B_{N}\right\} .
$$

And let $H^{\infty}=H^{\infty}\left(B_{N}\right)$ be the set of all bounded holomorphic functions on $B_{N}$. We denote by $B(A)\left(B\left(H^{\infty}\right)\right.$ resp.) the unit ball of $A\left(H^{\infty}\left(B_{N}\right)\right.$ resp.).

For $u, \varphi \in A$ with $\varphi \in S\left(B_{N}\right)$, recall that the composition operator $C_{\varphi}$ induced by $\varphi$ is defined by

$$
\left(C_{\varphi} f\right)(z)=f(\varphi(z))
$$

the multiplication operator induced by $u$ is defined by

$$
M_{u} f(z)=u(z) f(z)
$$

and the weighted composition operator $u C_{\varphi}$ induced by $\varphi$ and $u$ is defined by

$$
\left(u C_{\varphi} f\right)(z)=u(z) f(\varphi(z))
$$

for $z \in B_{N}$ and $f \in H\left(B_{N}\right)$. If let $u \equiv 1$, then $u C_{\varphi}=C_{\varphi}$; if let $\varphi=i d$, then $u C_{\varphi}=M_{u}$. It is clear that these operators are linear and $u C_{\varphi}$ is bounded on $A$. For some results in this topic see, for example, [1-6], and so on. 
Let $X$ be a Banach space of analytic functions, we write $C(X)$, for the space of composition operators on $X$ under the operator norm topology.

Moorhouse [7] considered the compactness of finite sum of composition operators on weighted Bergman spaces in the disk and gave a partial answer to the component structure of $C\left(A_{\alpha}^{2}\right)$. Kriete and Moorhouse [8] continued to study the finite linear combination of composition operators acting on the Hardy space or weighted Bergman spaces on the disk. Hosokawa and Ohno, [9], and [10], discussed the topological structures of the sets of composition operators and gave a characterization of compact difference on Bloch space in the unit disk. Fang and Zhou [11] then gave a characterization of compact difference between the Bloch space and the set of all bounded analytic functions on the unit polydisk. Fang and Zhou [12] also studied the compact differences of composition operators on the space of bounded analytic functions in polydisk. Hosokawa and co-workers [13] studied the topological components of the topological space of weighted composition operators on the space of bounded analytic functions on the open unit disk in the uniform operator topology. These results were extended to the setting of $H^{\infty}\left(B_{N}\right)$ by Toews [14], and independently by Gorkin and co-workers [15], and the setting of $H^{\infty}\left(D^{N}\right)$ by Fang and Zhou [12], where $D^{N}$ is the unit polydisk. Bonet and co-workers [16] discussed the same problem for the composition operator on the weighted Banach spaces of holomorphic functions in the unit disk, which was also extended to the unit polydisc by Wolf in [17]. The case of weighted composition operators on the above spaces was treated by Lindström and Wolf [18]. Ohno [19] studied the differences of weighted composition operators on the disk algebra.

Building on these foundations, we study the compactness of the sum of certain class of weighted composition operators on the unit ball algebra, and give the characterization of compact differences of two weighted composition operators on the ball algebra. The connectness of the topological space consisted of non-zero weighted composition operators on the unit ball algebra is also studied.

\section{The sum of weighted composition operators on $A$}

In this section, we will give necessary and sufficient conditions for the sum of several weighted composition operators to be compact on $A$.

Let $T$ be a bounded linear operator on a Banach space. Recall that $T$ is said to be compact if $T$ maps every bounded set into relatively compact one. And if $T$ is a compact operator, $T$ must map every weakly convergent sequence into norm convergent one, and a linear operator with that property is called to be completely continuous. In general, a completely continuous operator may not be always compact.

If $\varphi \in S\left(B_{N}\right) \cap A$, denote by $\Gamma_{\varphi}=\left\{\zeta \in \partial B_{N}:|\varphi(\zeta)|=1\right\}$. For $z, w \in B_{N}$, the involution automorphism between $z$ and $w$ is given by

$$
\Phi_{w}(z)=\frac{w-P_{w}(z)-s_{w} Q_{w}(z)}{1-<z, w>}
$$

where $P_{w}(z)=\frac{\langle z, w>}{<w, w>} w, Q_{w}(z)=z-\frac{<z, w>}{<w, w>} w$, and $s_{w}=\sqrt{1-|w|^{2}}$. The induced distance between $z$ and $w$ is defined as

$$
d_{\infty}(z, w)=\sup _{f \in B\left(H^{\infty}\right)}|f(z)-f(w)| .
$$


Pseudo-hyperbolic metric is defined by

$$
\rho(z, w)=\sup \left\{|f(z)|:\|f\|_{\infty}=1, f(w)=0\right\},
$$

and a theorem of Bear [20] gives that

$$
d_{\infty}(z, w)=\frac{2-2 \sqrt{1-\rho(z, w)^{2}}}{\rho(z, w)} .
$$

Since the pseudo-hyperbolic metric $\rho(z, w) \leq 1$ we have that

$$
\begin{aligned}
& 1-\rho(z, w)^{2} \leq \sqrt{1-\rho(z, w)^{2}} \\
\Rightarrow & 1-\sqrt{1-\rho(z, w)^{2}} \leq \rho(z, w)^{2} \\
\Rightarrow & d_{\infty}(z, w) \leq 2 \rho(z, w) .
\end{aligned}
$$

Toews [14] proved the following lemma:

Lemma 2.1. For any $z \in B_{N}$, we have

(a): $\rho(z ; w)=\left|\Phi_{w}(z)\right|$ for any $w \in B_{N}$, and

(b): $\{z: \rho(z, w)<\lambda\}=\Phi_{w}\left(\lambda B_{N}\right)$.

For $\zeta \in \partial B_{N}$ and $\alpha>1$, we define a Koranyi approach region by

$$
\Delta(\zeta, \alpha)=\left\{z \in B_{N}:|1-\langle z, \zeta\rangle|<\frac{\alpha}{2}\left(1-|z|^{2}\right)\right\} .
$$

Since $U \Delta(\zeta, \alpha)=\Delta(U \zeta, \alpha)$ for any unitary transformation $U$ in $\mathbb{C}^{N}$, we can concentrate on understanding $\Delta\left(e_{1}, \alpha\right)$, where $e_{1}=(1,0, \ldots, 0) \in \mathbb{C}^{N}$. Next lemma is crucial.

Lemma 2.2. For a real number $1<\alpha<2$ and $e_{1}=(1,0, \ldots, 0)$ in $\partial B_{N}$, if $\left\{w_{n}\right\}$ is an arbitrary sequence in the Koranyi approach region $\Delta\left(e_{1}, \alpha\right)$, then

$$
\left|\frac{\left|w_{n}\right|^{2}-\left\langle e_{1}, w_{n}\right\rangle}{1-\left\langle e_{1}, w_{n}\right\rangle}\right|>\frac{2}{\alpha}-1>0 .
$$

On the other hand, for every $\alpha>2$, we can find a sequence $\left\{w_{n}\right\} \subset \Delta\left(e_{1}, \alpha\right)$ and $w_{n} \rightarrow e_{1}$ as $n \rightarrow \infty$ satisfying

$$
\lim _{n \rightarrow \infty}\left|\frac{\left|w_{n}\right|^{2}-\left\langle e_{1}, w_{n}\right\rangle}{1-\left\langle e_{1}, w_{n}\right\rangle}\right|=0
$$

Proof. First, let $1<\alpha<2$. We have that

$$
\begin{aligned}
\left|\frac{\left|w_{n}\right|^{2}-\left\langle e_{1}, w_{n}\right\rangle}{1-\left\langle e_{1}, w_{n}\right\rangle}\right| & >\frac{2}{\alpha} \frac{\left.|| w_{n}\right|^{2}-\left\langle e_{1}, w_{n}\right\rangle \mid}{1-\left|w_{n}\right|^{2}} \\
& \geq \frac{2}{\alpha}\left(\frac{\left.|1-| w_{n}\right|^{2}|-| 1-\left\langle e_{1}, w_{n}\right\rangle \mid}{1-\left|w_{n}\right|^{2}}\right) \\
& >\frac{2}{\alpha}\left(1-\frac{\frac{\alpha}{2}\left(1-\left|w_{n}\right|^{2}\right)}{1-\left|w_{n}\right|^{2}}\right) \\
& =\frac{2}{\alpha}-1>0 .
\end{aligned}
$$


Suppose $\alpha>2$. Let $w_{n}=\left(r_{n}, w_{n}^{\prime}\right)$, where $r_{n}$ is real with $r_{n} \rightarrow 1^{-}$as $n \rightarrow \infty$ and $\left|w_{n}^{\prime}\right|=\sqrt{1-r_{n}}$. It is clear that for sufficient large $n$

$$
\begin{aligned}
& \left|1-\left\langle e_{1}, w_{n}\right\rangle\right|=1-r_{n}<\frac{\alpha}{2}\left(r_{n}-r_{n}^{2}\right) \\
= & \frac{\alpha}{2}\left(1-r_{n}^{2}-1+r_{n}\right)=\frac{\alpha}{2}\left(1-\left|w_{n}\right|^{2}\right) .
\end{aligned}
$$

This means that $\left\{w_{n}\right\} \subset \Delta\left(e_{1}, \alpha\right)$ for $\alpha>2$. Now we compute

$$
\lim _{n \rightarrow \infty}\left|\frac{\left|w_{n}\right|^{2}-\left\langle e_{1}, w_{n}\right\rangle}{1-\left\langle e_{1}, w_{n}\right\rangle}\right|=\lim _{n \rightarrow \infty} \frac{r_{n}^{2}+1-r_{n}-r_{n}}{1-r_{n}}=\lim _{n \rightarrow \infty}\left(1-r_{n}\right)=0 .
$$

$\square$

For $\varphi_{1}, \varphi_{2}, \ldots, \varphi_{p} \in S\left(B_{N}\right) \cap A$, we simplify the notations $\Gamma_{\varphi_{j}}$ and $C_{\varphi_{j}}$ by $\Gamma_{j}$ and $C_{j}$ respectively. It is obvious that each weighted composition operator is a bounded operator on the ball algebra.

For any $\zeta \in \bigcup_{j=1}^{p} \Gamma_{j}$, denote by $I(\zeta)=\left\{k \in\{1,2, \ldots, p\}: \zeta \in \Gamma_{k}\right\}$. If $k, j \in I(\zeta)$, define $k$ $\sim j$ whenever $\rho\left(\varphi_{j}\left(z_{n}\right), \varphi_{k}\left(z_{n}\right)\right) \rightarrow 0$ holds for every sequence $\left\{z_{n}\right\} \subset B_{N}$ converging to $\zeta$. It is clear that the relation $\sim$ is an equivalent relation on $I(\zeta)$. Denote by $I(\zeta) / \sim$ as the quotient set, and $I_{s}(\zeta)$ 's, which is called index sets of $\zeta$, as the elements of $I(\zeta) / \sim$. The lower index $s \in\{1, \ldots, p\}$ is given by the smallest number in $I s(\zeta)$.

Next, we are going to study the compactness of $\sum_{j=1}^{p} u_{j} C_{j}$ acting on the ball algebra. In fact, it is difficult to characterize the compactness of the sum of several arbitrary $u_{j} C_{j}$ 's. Here, we just consider the sum whose symbols are in a certain class of self mappings of $B_{N}$.

Our admissible symbols of self mappings of $B_{N}$ are constrained by the following condition: Let $\varphi \in S\left(B_{N}\right) \cap A$ and $\Gamma_{\varphi}$ as defined above. For every $\zeta \in \Gamma_{\varphi}$ and any arbitrary small $\varepsilon>0$, if there exists a Koranyi region $\Delta(\varphi(\zeta), \alpha)$ with $1<\alpha<2$ such that

$$
\left.{ }^{*}\right):(N(\varphi(\zeta), \varepsilon) \cap \Delta(\varphi(\zeta), \alpha)) \cap \varphi\left(B_{N}\right) \neq \varnothing
$$

where $N(\varphi(\zeta), \varepsilon)$ is the neighborhood of $\varphi(\zeta)$ with radii $\varepsilon$. We collect all self mappings of $B_{N}$ satisfied (*) and denote it by $A^{*}$.

Remark 2.3. If $\varphi \in S\left(B_{N}\right) \cap A$ and the closure of $\varphi\left(B_{N}\right)$ has no boundary contact with the unit sphere, we say that $\varphi$ satisfies condition (*) trivially, that is to say $\varphi \in A^{*}$.

The following theorem is our main theorem.

Theorem 2.4. Let $u_{j} \in A$ and $\varphi_{j} \in A^{*}$ for $j=1, \ldots, p$. Then $\sum_{j=1}^{p} u_{j} C_{j}$ is compact on $A$ if and only if and only if $\sum_{k \in I_{s}(\zeta)} u_{k}(\zeta)=0$ for every $\zeta \in \bigcup_{j=1}^{p} \Gamma_{j}$ and every index set $I_{s}(\zeta)$ of $\zeta$.

Proof. To prove the necessity, let $\zeta$ be an arbitrary point in $\bigcup_{j=1}^{p} \Gamma_{j}, I(\zeta)$ and $I_{s}(\zeta)$ 's are defined as above. We can find out a sequence $\left\{w_{n}\right\} \subset B_{N}$ satisfying the following properties: as $n \rightarrow \infty$,

(1): $w_{n} \rightarrow \zeta$

(2): $\varphi_{k}\left(w_{n}\right) \rightarrow \varphi_{k}(\zeta)$ with $k \in I_{s}(\zeta)$ and $k \neq s$;

(3): $\varphi_{l}\left(w_{n}\right) \rightarrow \varphi_{l}(\zeta)$ with $l \notin I_{s}(\zeta)$; 
(4): $\left\langle\Phi_{\varphi_{s}\left(w_{n}\right)}\left(\varphi_{s}(\zeta)\right), \varphi_{s}\left(w_{n}\right)\right\rangle \rightarrow \lambda \neq 0$

The item (4) holds since every $\varphi_{j} \in A^{*}$ and Lemma 2.2 guarantees the limit is non zero. From the item (3), we can choose the subsequences (also denoted by $\left\{w_{n}\right\}$ ) satisfying

(5): $\left\langle\Phi_{\varphi_{s}\left(w_{n}\right)}\left(\varphi_{l}\left(w_{n}\right)\right), \varphi_{s}\left(w_{n}\right)\right\rangle \rightarrow \sigma_{l s} \neq 0$.

For such a sequence $\left\{w_{n}\right\}$, define the functions

$$
\begin{aligned}
f_{n}(z)= & \left(\left\langle\Phi_{\varphi_{s}\left(w_{n}\right)}(z), \varphi_{s}\left(w_{n}\right)\right\rangle-1\right)\left(\left\langle\Phi_{\varphi_{s}\left(w_{n}\right)}(z), \varphi_{s}\left(w_{n}\right)\right\rangle-\lambda\right) \\
& \cdot \prod_{l \notin I_{s}(\zeta)}\left(\left\langle\Phi_{\varphi_{s}\left(w_{n}\right)}(z), \varphi_{s}\left(w_{n}\right)\right\rangle-\sigma_{l s}\right) .
\end{aligned}
$$

Then $f_{n} \in A$ and $\left\|f_{n}\right\|_{\infty} \leq 2^{p+1}$. We claim that $f_{n}(z) \rightarrow 0$ for all $z \in \overline{B_{N}}$ as $n \rightarrow \infty$. Indeed, if $z=\varphi_{s}(\zeta)$, the item (4) guarantees $f_{n}\left(\varphi_{s}(\zeta)\right) \rightarrow 0$; and if $z \neq \varphi_{s}(\zeta)$, we have

$$
\begin{aligned}
& \lim _{n \rightarrow \infty}\left\langle\Phi_{\varphi_{s}\left(w_{n}\right)}(z), \varphi_{s}\left(w_{n}\right)\right\rangle \\
= & \lim _{n \rightarrow \infty} \frac{\left\langle\varphi_{s}\left(w_{n}\right), \varphi_{s}\left(w_{n}\right)\right\rangle-\frac{\left\langle z, \varphi_{s}\left(w_{n}\right)\right\rangle}{\left\langle\varphi_{s}\left(w_{n}\right), \varphi_{s}\left(w_{n}\right)\right\rangle}\left\langle\varphi_{s}\left(w_{n}\right), \varphi_{s}\left(w_{n}\right)\right\rangle}{1-\left\langle z, \varphi_{s}\left(w_{n}\right)\right\rangle} \\
= & \lim _{n \rightarrow \infty} \frac{\left|\varphi_{s}\left(w_{n}\right)\right|^{2}-\left\langle z, \varphi_{s}\left(w_{n}\right)\right\rangle}{1-\left\langle z, \varphi_{s}\left(w_{n}\right)\right\rangle} \\
= & 1 .
\end{aligned}
$$

So $f_{n}$ converges weakly to 0 in $A$, thus $\left\|\sum_{j=1}^{p} u_{j} C_{j} f_{n}\right\|_{\infty} \rightarrow 0$ as $n \rightarrow \infty$.

On the other hand,

$$
\begin{aligned}
& \left.|| \sum_{j=1}^{p} u_{j} C_{j} f_{n}||\right|_{\infty} \geq\left|\sum_{j=1}^{p} u_{j}\left(w_{n}\right) f_{n}\left(\varphi_{j}\left(w_{n}\right)\right)\right| \\
\geq & \left|\sum_{k \in I_{s}(\zeta)} u_{k}\left(w_{n}\right)\right||\lambda| \prod_{l \notin I_{s}(\zeta)} \sigma_{l s}-\sum_{l \notin I_{s}(\zeta)}\left|u_{l}\left(w_{n}\right)\right|\left|f_{n}\left(\varphi_{l}\left(w_{n}\right)\right)\right| \\
& -\sum_{k \in I_{s}(\zeta) \backslash\{s\}}\left|u_{k}\left(w_{n}\right)\right|\left|f_{n}\left(\varphi_{s}\left(w_{n}\right)\right)-f_{n}\left(\varphi_{k}\left(w_{n}\right)\right)\right| \\
\geq & \left|\sum_{k \in I_{s}(\zeta)} u_{k}\left(w_{n}\right)\right||\lambda| \prod_{l \notin I_{s}(\zeta)} \sigma_{l s}-\sum_{l \notin I_{s}(\zeta)}\left|u_{l}\left(w_{n}\right)\right|\left|f_{n}\left(\varphi_{l}\left(w_{n}\right)\right)\right| \\
& -2 \sum_{k \in I_{s}(\zeta) \backslash\{s\}}\left\|u_{k}\right\|_{\infty}\left\|f_{n}\right\|_{\infty} \rho\left(\varphi_{s}\left(w_{n}\right), \varphi_{k}\left(w_{n}\right)\right) .
\end{aligned}
$$

We have that $\rho\left(\varphi_{s}\left(w_{n}\right), \varphi_{k}\left(w_{n}\right)\right) \rightarrow 0$ for every $k \in I_{s}(\zeta) \backslash\{s\}$. And from the item (5), we know that $\left|f_{n}\left(\varphi_{l}\left(w_{n}\right)\right)\right| \rightarrow 0$ for every $l \notin I_{s}(\zeta)$. Hence we have

$$
\sum_{k \in I_{s}(\zeta)} u_{k}(\zeta)=0
$$

To verify the sufficiency, we cannot use the "weak convergence theorem" (Proposition 3.11 of [21]), because the unit ball algebra is not closed in the compact open topology. Here, we use the definition of compact operator to illustrate that the sum of 
weighted composition operators is compact. Let $f_{n} \in A$ and $\left\|f_{n}\right\|_{\infty}=1$. By the normal family argument, there exists a subsequence $\left\{f_{n_{k}}\right\}$ of $\left\{f_{n}\right\}$ and a function $g$ analytic on $B_{N}$ such that $f_{n_{k}}$ converges to $g$ uniformly on compact subsets of $B_{N}$. Here, we have

$$
\sup _{z \in B_{N}}|g(z)| \leq 1 \text {. }
$$

Now define a function $G$ on $\overline{B_{N}}$ by setting

$$
G(z)= \begin{cases}\sum_{l \notin I(z)} u_{l} C_{l} g(z) & z \in \bigcup_{j=1}^{p} \Gamma_{j} ; \\ \sum_{j=1}^{p} u_{j} C_{j} g(z) & \text { otherwise. }\end{cases}
$$

Next, we just need to show that $G$ is continuous on $\overline{B_{N}}$, and $\sum_{j=1}^{p} u_{j} C_{j} f_{n_{k}}$ converges uniformly to $G$ on $\overline{B_{N}}$. In the following proof, we simplify the subsequence $f_{n_{k}}$ by $f_{n}$.

We first prove that $G$ is continuous on $\overline{B_{N}}$. Indeed, it is obvious that $G$ is continuous on $\overline{B_{N}} \backslash \bigcup_{j=1}^{p} \Gamma_{j}$.

For $\zeta \in \bigcup_{j=1}^{p} \Gamma_{j}$, let $I_{s}(\zeta)$ 's be its index sets and $I(\zeta)=\bigcup_{s} I_{s}(\zeta)$. Suppose that $\left\{z_{n}\right\}$ be a sequence in $B_{N}$ converging to $\zeta$ such that $\varphi_{j}\left(z_{n}\right) \rightarrow \varphi_{j}(\zeta) j=1, \ldots, p$. So for each $I_{s}(\zeta)$.

- $\rho\left(\varphi_{k}\left(z_{n}\right), \varphi_{s}\left(z_{n}\right)\right) \rightarrow 0$ where $k \in I_{s}(\zeta)$, and

- $\rho\left(\varphi_{l}\left(z_{n}\right), \varphi_{s}\left(z_{n}\right)\right) \nrightarrow 0$ where $l \notin I(\zeta)$.

Then, we compute

$$
\begin{aligned}
& \left|\sum_{j=1}^{p} u_{j} C_{j} g\left(z_{n}\right)-\sum_{l \notin \in I(\zeta)} u_{l} C_{l} g(\zeta)\right| \\
\leq & \sum_{s}\left(\|g\|_{\infty}\left|\sum_{k \in I_{s}(\zeta)} u_{k}\left(z_{n}\right)\right|+2 \sum_{k \in I_{s}(\zeta) \backslash\{s\}}\left\|u_{k}\right\|_{\infty}\|g\|_{\infty} \rho\left(\varphi_{s}\left(z_{n}\right), \varphi_{k}\left(z_{n}\right)\right)\right) \\
& +\left|\sum_{l \notin I(\zeta)}\left(u_{l} C_{l} g\left(z_{n}\right)-u_{l} C_{l} g(\zeta)\right)\right| .
\end{aligned}
$$

So, we have $\lim _{z_{n} \rightarrow \zeta} \sum_{j=1}^{p} u_{j} C_{j} g\left(z_{n}\right)=G(\zeta)$, and this holds for every $\zeta \in \bigcup_{j=1}^{p} \Gamma_{j}$. Thus $G$ is continuous on $\overline{B_{N}}$.

Next, we shall show that $\sum_{j=1}^{p} u_{j} C_{j} f_{n}$ converges uniformly to $G$ on $\overline{B_{N}}$. Suppose not, and we may assume that, for $\varepsilon>0,\left\|\sum_{j=1}^{p} u_{j} C_{j} f_{n}-G\right\|_{\infty}>\varepsilon>0$. Then there exists a sequence $\left\{z_{n}\right\} \subset B_{N}$ such that

$$
\left|\sum_{j=1}^{p} u_{j} C_{j} f_{n}\left(z_{n}\right)-G\left(z_{n}\right)\right|>\varepsilon \text { for every } n .
$$

This implies that $\max _{j}\left\{\left|\varphi_{j}\left(z_{n}\right)\right|\right\} \rightarrow 1$ as $n \rightarrow \infty$. Here we may assume that $z_{n} \rightarrow \zeta \in$ $\partial B_{N}$, and define $I(\zeta), I_{s}(\zeta)$ as before. Similarly as the analysis above, we have that

- $\rho\left(\varphi_{k}\left(z_{n}\right), \varphi_{s}\left(z_{n}\right)\right) \rightarrow 0$ where $k \in I_{s}(\zeta)$,

- $\rho\left(\varphi_{l}\left(z_{n}\right) ; \varphi_{s}\left(z_{n}\right)\right) \nrightarrow 0$ where $l \notin I_{s}(\zeta)$. 
Hence

$$
\begin{aligned}
& \left|\sum_{j=1}^{p}\left(u_{j} C_{j}\right) f_{n}\left(z_{n}\right)-G\left(z_{n}\right)\right| \\
\leq & \sum_{s}\left(\left\|f_{n}\right\|_{\infty}\left|\sum_{k \in I_{s}(\zeta)} u_{k}\left(z_{n}\right)\right|+2 \sum_{k \in I_{s}(\zeta) \backslash\{s\}}\left\|u_{k}\right\|_{\infty}\left\|f_{n}\right\|_{\infty} \rho\left(\varphi_{s}\left(z_{n}\right), \varphi_{k}\left(z_{n}\right)\right)\right) \\
& +\left|\sum_{l \notin I(\zeta)} u_{l}\left(z_{n}\right)\left[f_{n}\left(\varphi_{l}\left(z_{n}\right)\right)-g\left(\varphi_{l}\left(z_{n}\right)\right)\right]\right| .
\end{aligned}
$$

When $l \notin I(\zeta)$, note that the limit of $\left|\varphi_{l}\left(z_{n}\right)\right|$ is strictly less than 1 , and $f_{n}$ converges to $g$ on compact subsets of $B_{N}$, thus $f_{n}\left(\varphi_{l}\left(z_{n}\right)\right)-g\left(\varphi_{l}\left(z_{n}\right)\right) \rightarrow 0$. By the preliminary conditions we know that $\left|\sum_{k \in I_{s}(\zeta)} u_{k}(\zeta)\right|=0$ for each index sets $I_{s}(\zeta)$. Together with the above two items, we have $\sum_{j=1}^{p} u_{j} C_{j} f_{n}\left(z_{n}\right)-G\left(z_{n}\right) \rightarrow 0$. This fact contradicts (2.1). Thus, we have proved the sufficient condition.

As a corollary of the sum theorem, we state the characterization of the compact differences of two weighted composition operators on $A$.

Theorem 2.5 Let $u, v \in A$ and $\varphi, \psi \in A^{*}$. Then $u C_{\varphi}-v C_{\psi}$ is compact on $A$ if and only if the following three conditions hold:

(a): If $\zeta \in \Gamma_{\varphi}$ and $\lim _{z \rightarrow \zeta} \rho(\varphi(z), \psi(z)) \neq 0$, then $u(\zeta)=0$;

(b): If $\zeta \in \Gamma_{\psi}$ and $\lim _{z \rightarrow \zeta} \rho(\varphi(z), \psi(z)) \neq 0$, then $v(\zeta)=0$;

(c): If $\zeta \in \Gamma_{\varphi} \cap \Gamma_{\psi}$, then $u(\zeta)=v(\zeta)$.

Corollary 2.6 Let $u \in A$ and $\varphi \in A^{*}$, the associated weighted composition operator $u C_{\varphi}$ act compactly on $A$ if and only if $u(\zeta)=0$ for every $\zeta \in \Gamma_{\varphi}$.

Proof. Just set $v(z)=-u(z)$ and $\psi(z)=\varphi(z)$ in the proof of Theorem 2.4. $\square$

Corollary 2.7 Let $\varphi, \psi \in A^{*}$. Then the following conditions are equivalent:

(i): $C_{\varphi}-C_{\psi}$ is compact on $A$;

(ii): $C_{\varphi}-C_{\psi}$ is completely continuous on $A$

(iii): $\Gamma_{\varphi}=\Gamma_{\psi}$.

Now, we illustrate Theorem 2.4 with the following example.

Example. If $\varphi_{j}(j=1, \ldots, 5)$ are analytic self maps of $B_{N}$ with following formulas:

$$
\begin{aligned}
\varphi_{1}\left(z_{1}, \ldots, z_{N}\right) & =\left(\frac{z_{1}+1}{2}, \frac{z_{2}}{2}, \ldots, \frac{z_{N}}{2}\right), \\
\varphi_{2}\left(z_{1}, \ldots, z_{N}\right) & =\left(\frac{z_{2}}{2}, \frac{z_{1}+1}{2}, \ldots, \frac{z_{N}}{2}\right), \\
\varphi_{3}\left(z_{1}, \ldots, z_{N}\right) & =\left(\frac{z_{1}+3}{4}, \frac{z_{2}}{4}, \ldots, \frac{z_{N}}{4}\right), \\
\varphi_{4}\left(z_{1}, \ldots, z_{N}\right) & =\left(\frac{z_{1}^{2}+1}{2}, 0, \ldots, 0\right), \\
\varphi_{5}\left(z_{1}, \ldots, z_{N}\right) & =\left(\frac{z_{1}}{2}, \frac{z_{2}}{2}, \ldots, \frac{z_{N}}{2}\right),
\end{aligned}
$$


and

$$
\begin{gathered}
u_{1}\left(z_{1}, \ldots, z_{N}\right)=2 z_{1}, \quad u_{2}\left(z_{1}, \ldots, z_{2}\right)=z_{2}+\cdots+z_{N} \\
u_{3}\left(z_{1}, \ldots, z_{N}\right)=-3 z_{1}, \quad u_{4}\left(z_{1}, \ldots, z_{N}\right)=\left(z_{1}+1\right) / 2, \quad u_{5} \equiv 1 .
\end{gathered}
$$

Then, $\sum_{j=1}^{5} u_{j} C_{j}$ is a compact operator on $A$.

Proof. First, note that $\Gamma_{1}=\{(1,0, \ldots, 0)\}=\left\{e_{1}\right\}, \Gamma_{2}=\left\{e_{1}\right\}, \Gamma_{3}=\left\{e_{1}\right\}, \Gamma_{4}=\left\{ \pm e_{1}\right\}, \Gamma_{2}=\varnothing$. And $\varphi_{1}\left(e_{1}\right)=\varphi_{3}\left(e_{1}\right)=e_{1}, \varphi_{2}\left(e_{1}\right)=(0,1,0, \ldots, 0)=\left(e_{2}\right), \varphi_{4}\left( \pm e_{1}\right)=e_{1}$. Then, $\varphi_{j} \in A^{*}$ for each $j=1, \ldots, 5$. Take $\varphi_{1}$ for example, the sequence $\{(1-1 / n, 0, \ldots, 0)\}$ converges to $\varphi$ $\left(e_{1}\right)$ radially, and they have pre-images $\{(1-2 / n, 0, \ldots, 0)\}$ of $\varphi_{1}$ in $B_{N}$. Hence, $\varphi_{1}$ satisfies $\left(^{*}\right)$. Then, we have $I_{1}\left(e_{1}\right)=\{1,3,4\}, I_{2}\left(e_{1}\right)=\{2\}$ and $I\left(-e_{1}\right)=I_{4}\left(-e_{1}\right)=\{4\}$. Compute that

$$
\begin{aligned}
& I_{1}\left(e_{1}\right): u_{1}\left(e_{1}\right)+u_{3}\left(e_{1}\right)+u_{4}\left(e_{1}\right)=2-3+1=0, \\
& I_{2}\left(e_{1}\right): u_{2}\left(e_{1}\right)=0, \\
& I\left(-e_{1}\right): u_{4}\left(-e_{1}\right)=0 .
\end{aligned}
$$

By Theorem 2.4, $\sum_{j=1}^{5} u_{j} C_{j}$ is compact on $A$. $\square$

\section{Connectness of weighted composition operators on $\boldsymbol{A}$}

Shapiro and Sundberg [22] first discovered the relationship between compact differences and topological structure of the collection of composition operators on Hardy space. In this section, we will investigate the connectness of the topological space consisted of all weighted composition operators act on the unit ball algebra, and we use the notion $\mathcal{C}(A)$ to represent that topological space. It is trivial that

\section{$\mathcal{C}(A)$ is a connected topological space.}

Because we can connect any $u C_{\varphi}$ and 0 (the weighted composition operator $u C_{\varphi}$ when $u \equiv 0)$ by the continuous path $T_{t}:[0,1] \rightarrow \mathcal{C}(A)$ defined by $t \mapsto t u C_{\varphi}$. Indeed, for many function spaces, we denote by $S$ generally, the weighted composition operators topological space $\mathcal{C}(S)$ is connected by the same path constructed above. So, we are interested in the connectness of the non-zero weighted composition operators topological space, and here we denote by $\mathcal{C}_{w}(S)$. In this setting, the connectness of $\mathcal{C}_{w}(S)$ depends on the function space $S$.

Theorem $3.1 \mathcal{C}_{w}(A)$ is also a connected topological space.

Proof. Let distinct $u C_{\varphi}, v C_{\psi} \in \mathcal{C}_{w}(A)$. We construct a path

$$
T(t):[0,1] \rightarrow \mathcal{C}_{w}(A) \text { by } t \mapsto u_{t} C_{\varphi_{t}}
$$

where $u_{t}(z)=t u(z)+(1-t) v(z)$ and $\varphi_{t}(z)=t \varphi(z)+(1-t) \psi(z)$. To avoid $u_{t}=0$ and $\varphi_{t}$ $=0$ for some $t \in[0,1]$, we first assume that

(1): $v \neq \theta u$ for every $\theta<0$;

(2): $\psi \neq \kappa \varphi$ for every $\kappa<0$ with $\kappa \varphi \in S\left(B_{N}\right)$, where $\psi=\left(\psi_{1}, \ldots, \psi_{N}\right)$ and $\varphi=\left(\varphi_{1}, \ldots\right.$, $\left.\varphi_{N}\right)$.

We are going to prove that $T(t)$ is a continuous path which connects $u C_{\varphi}$ and $\nu C_{\psi}$, 
and here we simply denote by $C_{t}=C_{\varphi_{t}}$. For any $s, t \in[0,1]$,

$$
\left\|u_{t} C_{t}-v_{t} C_{s}\right\| \leq\left\|u_{t}\left(C_{t}-C_{s}\right)\right\|+\left\|\left(u_{t}-u_{s}\right) C_{s}\right\| .
$$

The second term:

$$
\left\|\left(u_{t}-u_{s}\right) C_{s}\right\| \leq|t-s| \cdot\|u+v\|_{\infty}\left\|C_{s}\right\| \leq|t-s| \cdot\|u+v\|_{\infty} .
$$

So, it converges to 0 as $s \rightarrow t$.

The first term:

$$
\begin{aligned}
& \left\|u_{t}\left(C_{t}-C_{s}\right)\right\| \\
\leq & \left\|u_{t}\right\|_{\infty}\left\|C_{t}-C_{s}\right\| \\
= & \left\|u_{t}\right\|_{\infty} \sup _{f \in B(A)} \sup _{z \in B_{N}}\left|\left(C_{t}-C_{s}\right) f(z)\right| \\
\leq & \left\|u_{t}\right\|_{\infty} \sup _{f \in B\left(H^{\infty}\right.} \sup _{z \in B_{N}}\left|f\left(\varphi_{t}(z)\right)-f\left(\varphi_{s}(z)\right)\right| \\
= & \left\|u_{t}\right\|_{\infty} \sup _{z \in B_{N}} \sup _{f \in B\left(H^{\infty}\right)}\left|f\left(\varphi_{t}(z)\right)-f\left(\varphi_{s}(z)\right)\right| \\
\leq & 2\left\|u_{t}\right\|_{\infty} \sup _{z \in B_{N}} \rho\left(\varphi_{t}(z), \varphi_{s}(z)\right) .
\end{aligned}
$$

So, it converges to 0 as $s \rightarrow t$, since $\varphi_{t}, \varphi_{s} \in A$ (See Lemma 6 in [14]). Here, we obtain that $\{T(t)\}$ is a continuous path which connects any different $u C_{\varphi}$ and $\nu C_{\psi}$ in $\mathcal{C}_{w}(A)$.

Next, for any given $u C_{\varphi} \in \mathcal{C}_{w}(A)$, we need to find out continuous pathes from $u C_{\varphi}$ to either $\theta u C_{\varphi}$ or $u C_{\kappa \varphi}$ where $\theta<0$ and $\kappa<0$ with $\kappa \varphi \in S\left(B_{N}\right) \cap A$.

It is obvious that $-u C_{\varphi}$ and $\theta u C_{\varphi}$ can be connected. Then, we construct

$$
R(t)=e^{i \pi t} u \cdot C_{\varphi}:[0,1] \rightarrow \mathcal{C}_{w}(A)
$$

to connect $u C_{\varphi}$ and $-u C_{\varphi}$ continuously: for $s, t \in[0,1]$,

$$
\left\|e^{i \pi t} u C_{\varphi}-e^{i \pi s} u C_{\varphi}\right\| \leq\left|e^{i \pi t}-e^{i \pi s}\right|\|u\|_{\infty}\left\|C_{\varphi}\right\| \leq \pi|t-s|\|u\|_{\infty}\left\|C_{\varphi}\right\| \rightarrow 0
$$

as $|t-s| \rightarrow 0$. Thus, $\theta u C_{\varphi}$, with any $\theta<0$, can be connected continuously with $u C_{\varphi}$.

Similarly as the analysis above, we note that $-\varphi$ cannot be equal to $\kappa \varphi$ multiplied by any negative real numbers. Thus, $u C_{-\varphi}$ and $u C_{\kappa \varphi}$ can be connected. To connect $u C_{-\varphi}$ and $u C_{\varphi}$, we put $\mu=i \varphi \in S\left(B_{N}\right)$, and

$$
\begin{gathered}
Y_{1}(t)=u C_{t \varphi+(1-t) \mu}:[0,1] \rightarrow \mathcal{C}_{w}(A) \\
Y_{2}(t)=u C_{t(-\varphi)+(1-t) \mu}:[0,1] \rightarrow \mathcal{C}_{w}(A)
\end{gathered}
$$

By the same method of computation as in (3.1), $Y_{1}(t)$ and $Y_{2}(t)$ can be shown to be two continuous pathes in $\mathcal{C}_{w}(A)$ from $u C_{\varphi}$ and $u C_{-\varphi}$ to $u C_{\mu}$.

Thus, the space $\mathcal{C}_{w}(A)$ is a connected topological space.

Corollary 3.2 The topological space consisting of composition operators acting on the unit ball algebra is connected.

From the last result, we know that two weighted composition operators can be in the same component in $\mathcal{C}_{w}(A)$ without compact differences. 


\section{Competing interests}

The authors declare that they have no competing interests.

Received: 8 April 2011 Accepted: 2 September 2011 Published: 2 September 2011

\section{References}

1. Xiao, J: Composition operators associated with Bloch-type spaces. Complex Variables. 46, 109-121 (2001)

2. Zhu, KH: Operator Theory in Function Spaces. Marcel Dekker. Inc., New York (1990)

3. Zhu, KH: Spaces of Holomorphic Functions in the Unit Ball. Grad Texts in Math. Springer, New York (2005)

4. Zhou, ZH, Chen, RY: Weighted composition operators fom $F(p, q, s)$ to Bloch type spaces. Inter J Math. 19(8), 899-926 (2008). doi:10.1142/S0129167X08004984

5. Zhou, ZH, Liu, Y: The essential norms of composition operators between generalized Bloch spaces in the polydisc and its applications. J Inequ Appl 2006, 22 (2006). (Article ID 90742)

6. Zhou, ZH, Shi, JH: Compactness of composition operators on the Bloch space in classical bounded symmetric domains. Michigan Math J. 50, 381-405 (2002). doi:10.1307/mmj/1028575740

7. Moorhouse, J: Compact differences of composition operators. J Funct Anal. 219, 70-92 (2005). doi:10.1016/j. jfa.2004.01.012

8. Kriete, T, Moorhouse, J: Linear relations in the Calkin algebra for composition operators. Trans Am Math Soc. 359, 2915-2944 (2007). doi:10.1090/50002-9947-07-04166-9

9. Hosokawa, T, Ohno, S: Topological structures of the sets of composition operators on the Bloch spaces. J Math Anal Appl. 314, 736-748 (2006). doi:10.1016/j.jmaa.2005.04.080

10. Hosokawa, T, Ohno, S: Differences of composition operators on the Bloch spaces. J Oper Theory. 57, 229-242 (2007)

11. Fang, ZS, Zhou, ZH: Differences of composition operators on the Bloch space in the polydisc. Bull Aust Math Soc. 79(3), 465-471 (2009). doi:10.1017/50004972709000045

12. Fang, ZS, Zhou, ZH: Differences of composition operators on the space of bounded analytic functions in the polydisc. Abstr Appl Anal 2008, 10 (2008). (Article ID 983132)

13. Hosokawa, T, Izuchi, K, Ohno, S: Topological structure of the space of weighted composition operators on $H^{\circ}$. Integr Equ Oper Theory. 53, 509-526 (2005). doi:10.1007/s00020-004-1337-1

14. Toews, C: Topological components of the set of composition operators on $H^{\infty}\left(B_{N}\right)$. Integr Equ Oper Theory. 48, 265-280 (2004). doi:10.1007/s00020-002-1180-1

15. Gorkin, P, Mortini, R, Suarez, D: Homotopic composition operators on $H^{\circ}\left(B^{n}\right)$, function spaces, Edwardsville, IL (2002) 177C188; Contemporary Mathematics, 328. Am Math Soc, Providence, RI. (2003)

16. Bonet, J, Lindström, M, Wolf, E: Differences of composition operators between weighted Banach spaces of holomorphic functions. J Aust Math Soc. 84, 9-20 (2008)

17. Wolf, E: Differences of composition operators between weighted Banach spaces of holomorphic functions on the unit polydisk. Result Math. 51, 361-372 (2008). doi:10.1007/s00025-007-0283-z

18. Lindström, M, Wolf, E: Essential norm of the difference of weighted composition operators. Monatsh Math. 153, 133-143 (2008). doi:10.1007/s00605-007-0493-1

19. Ohno, S: Differences of weighted composition operators on the disk algebra. Bull Belg Math Soc Simon Stevin. 17, 101-107 (2010)

20. Bear, H: Lectures on Gleason parts, Lecture Note in Mathematics 121. Springer, Berlin and New York. (1970)

21. Cowen, CC, MacCluer, BD: Composition Operators on Spaces of Analytic Functions. CRC Press, Boca Raton. (1995)

22. Shapiro, JH, Sundberg, C: Isolation amongst the composition operators. Pacific J Math. 145, 117-152 (1990)

doi:10.1186/1029-242X-2011-45

Cite this article as: Tong and Zhou: The compactness of the sum of weighted composition operators on the ball algebra. Journal of Inequalities and Applications 2011 2011:45.

\section{Submit your manuscript to a SpringerOpen ${ }^{\circ}$ journal and benefit from:}

- Convenient online submission

Rigorous peer review

- Immediate publication on acceptance

- Open access: articles freely available online

- High visibility within the field

- Retaining the copyright to your article

Submit your next manuscript at $\$$ springeropen.com 